\title{
"Ultra-mixing": a Simple and Effective Method to Obtain Controlled and Stable Dispersions of Graphene Oxide in Cell Culture Media
}

\author{
Giacomo Reina, ${ }^{a *}$ Amalia Ruiz, ${ }^{a}$ Diane Murera, ${ }^{a}$ Yuta Nishina, ${ }^{\mathrm{b}, \mathrm{c}}$ Alberto Bianco ${ }^{\mathrm{a} *}$ \\ a University of Strasbourg, CNRS, Immunology, Immunopathology and Therapeutic \\ Chemistry, UPR 3572, 67000 Strasbourg, France \\ ${ }^{b}$ Graduate School of Natural Science and Technology, Okayama University, \\ Tsushimanaka, Kita-ku, Okayama 700-8530, Japan \\ ${ }^{\mathrm{C}}$ Research Core for Interdisciplinary Sciences, Okayama University, Tsushimanaka, Kita- \\ ku, Okayama 700-8530, Japan
}

Corresponding author: a.bianco@ibmc-cnrs.unistra.fr; g.reina@ibmc-cnrs.unistra.fr

Keywords: Carbon nanomaterials, quantum dots, complexes, corona, dispersibility 


\begin{abstract}
The last decade has seen an increase in the application of graphene oxide (GO) in the biomedical field. GO has been successfully exploited for its ability to deliver many kinds of drugs into target cells. However, GO toxicity assessment is still controversial. Several studies demonstrated that GO protein coating is crucial to alleviate the material's toxicity. Besides, coronation leads to the formation of big agglomerates, reducing the cellular uptake of the material and thus its therapeutic efficiency. In this work, we propose a simple and efficient method based on rapid (Ultra-Turrax, UT) mixing to control protein corona formation. Using UT protocol, we were able to reduce GO agglomeration in the presence of proteins and to obtain stable GO dispersions in cell culture media. By labelling GO with luminescent nanoparticles (QDs), we studied the GO internalization kinetic and efficiency. Comparing the "classic" and the UT protocols, we found that the latter allows a faster and more efficient internalization both in macrophages and HeLa cells without affecting cell viability. We believe that the use of UT protocol will be interesting and suitable for the preparation of next generation GO-based drug delivery platforms.
\end{abstract}




\section{Introduction}

The last decade has seen a remarkable growth of carbon nanomaterial applications in the biomedical field. ${ }^{1}$ In particular, the graphene family nanomaterials are promising to tear down the current limits in the biomedical fields by proposing innovative solutions for biodetection, tissue engineering, and drug and gene delivery. ${ }^{2}$ For the latter application, graphene oxide has certainly become the most studied nanomaterial. $\mathrm{GO}$ is produced by the exfoliation process of graphite under strong oxidative conditions. Chemically speaking, $\mathrm{GO}$ is composed by a single-layer of carbon (like graphene) where 40 to $60 \%$ of the $\mathrm{C}$ atoms are bound to oxygen. The oxidation process enriches the nanosheets with different highly polar oxygen functional groups (i.e. epoxides, alcohols and carboxylic acids) that render GO extremely stable in water and in many other polar solvents. ${ }^{3}$ Moreover, GO production have been standardized with treatments to ensure low contamination risks (organic and inorganic), tailored size and high reproducibility. ${ }^{2}$ Several studies have demonstrated the ability of GO to penetrate cell membranes and release small drugs, genetic materials, or macromolecules. ${ }^{2,4}$ Additionally, GO can be functionalized with different molecules such as dispersants, imaging agents, targeting and therapeutic molecules, making this material a perfect platform for theragnostic and multimodal applications. $^{2,4}$

However, several issues still need to be addressed. Currently, application of GO in the biomedical field is ruled by the dichotomy between efficiency of the treatment (in vivo or in vitro) and the GO toxicity. Before any GO-based therapy reaches the clinical stage, the evaluation of risks over benefits must be carefully analyzed. Regarding the risks, it has been observed that concentration, size, exposure method and time strongly affect the GO toxicity, often leading to controversial results. ${ }^{5} \mathrm{~A}$ crucial step in understanding and eventually modulating the $\mathrm{GO}$ toxicity is related to its interaction with proteins. ${ }^{6}$ Indeed, each GO sheet is able to stably load a huge amount (from 2 to 12 times its weight) of proteins onto its surface. ${ }^{7,8}$ This interaction strongly depends on protein structure and is due to a mixture of different contributions. ${ }^{7,8}$ Non-covalent interactions are driven by enthalpy with a main contribution of low polar interactions happening between $\mathrm{C} \mathrm{sp}^{2}$ aromatic domains and the lipophilic residues. ${ }^{9}$ Besides, covalent reactions can also occur on GO surface. In the latter case, radical addition ${ }^{10,11}$ and epoxide nucleophilic ring opening are the most probable reactions that could happen between serum proteins and GO sheets. Despite the unclear GO-protein coronation, it is known that this process is very short (5-10 minutes) and strongly alters the protein secondary structure. ${ }^{8}$ In particular, it 
has been observed that the interaction with GO leads to the conversion of $\alpha$-helix into $\beta$ sheet, causing the subsequent agglomeration into the cell culture media. ${ }^{8}$ Interaction with proteins must therefore be carefully evaluated for the preparation of GO in drug delivery applications. Indeed, the formation of covalent or non-covalent protein corona on the nanostructures irreversibly alters their dispersibility, cell uptake and most importantly the pharmacokinetics and pharmacodynamics. ${ }^{5}$ Most of the drug administration pathways involve local or intravenous injection, where the nanocarriers immediately encounter plasmatic proteins leading to the corona formation. Different studies demonstrated that GO cytotoxicity can be alleviated by the first interaction with serum proteins. ${ }^{6,11,12}$ The corona formation can mitigate the material cytotoxicity but it can also affect the colloidal stability of the formulation, due to the formation of large agglomerates and consequently slowing down or reducing the cell uptake. ${ }^{5}$ Although numerous in vitro toxicity studies using GObased materials have been performed, to date these studies have not resulted in the creation of a standardized protocol applicable to many of the new candidates under development for biomedical applications.

In this work we propose a method to control the corona formation by reducing the degree of flake agglomeration. This is achieved by premixing the $\mathrm{GO}$ with the serum proteins using Ultra-Turrax (UT), a disperser based on the rotor-stator technology able to disperse the material via a high circumferential speed. We compared the UT technique with the "classic" mixing of the material with the biological media. Aiming to follow the nanomaterial uptake, we prepared GO functionalized with luminescent nanocrystals. We used HeLa cells, as both nonphagocytic epithelial and cancer cell model, and Raw 264.7 macrophages as immune and phagocytic cell model. These two cell lines represent two important models for general in vitro evaluation of cytotoxicity of nanomaterials for biomedical applications. By comparing the two methods, we found that the UT allows the preparation of a dispersion with smaller GO agglomerates and higher colloidal stability. This allows us to increase the speed and quantity of the GO taken up into both cell lines. In addition, the UT treatment improves cell viability in Raw 264.7 macrophages incubated with the functionalized GO, suggesting that this could be a crucial step in the safety studies of GO-based formulations for drug delivery. 


\section{Experimental}

\subsection{Materials}

Graphene oxide was synthesized following the modified method by Prof. Nishina ${ }^{13}$ and obtained as an aqueous dispersion at $3 \mathrm{mg} / \mathrm{ml}$ concentration. All the chemicals were purchased from Sigma Aldrich. The solvents were obtained from commercial suppliers and used without purification. Water was purified using a Millipore filter system MilliQ ${ }^{\circledR}$ and free endotoxin Polisseur Biopak ${ }^{\circledR}$. When stated, suspensions were sonicated in a water bath (20 W, $40 \mathrm{kHz})$.

\subsection{Instruments}

TEM analysis were carried out with Hitachi 7500 transmission electron microscope (Hitachi High Technologies Corporation, Tokyo, Japan) equipped with an AMT Hamamatsu digital camera (Hamamatsu Photonics, Hamamatsu City, Japan). Fluorescence steady state spectra were recorded via Fluorolog FL3-22 (HORIBA JobinYvon) using swig xenon 450 W lamp. Lifetime measurements were recorded using light-emitting diodes (NanoLED) exciting at $460 \mathrm{~nm}$ at a fixed emission of $710 \mathrm{~nm}$. The GO-QD and QDs were analyzed at $10 \mu \mathrm{g} / \mathrm{ml}$ concentration. For the dispersion Ultra-Turrax (IKA) t10 was used.

XPS analyses were performed on a Thermo Scientific K-Alpha X-ray photoelectron spectrometer with a basic chamber pressure of $10^{-8}-10^{-9}$ bar with an anode using $\mathrm{Al} \mathrm{Ka}$ radiation $(\mathrm{hv}=1486.6 \mathrm{eV})$. The samples were analyzed as a powder. Spots size of 400 $\mu \mathrm{m}$ were used. The survey spectra are average of 10 scans with a pass energy of 200.00 $\mathrm{eV}$ and a step size of $1 \mathrm{eV}$. An ion gun was turned on during analysis. For each sample the analysis was repeated three times.

Confocal images have been carried out with a Microscope Zeiss Axio Observer Z1 Confocal Spinning disk equipped with 63 or $100 \mathrm{X}$ oil objective. Z-staking was recorded with $0.3 \mu \mathrm{m}$ interplanar distance. The fluorescence signal from Cell-Mask was obtained using $488 \mathrm{~nm}$ laser excitation and recording in the green channel (505-555 nm) while GOQD, was recorded with using $488 \mathrm{~nm}$ laser excitation, in the far-red channel (665-715 $\mathrm{nm}$ ). Images have been then treated with FIJI software.

\subsection{Methods}

ZnS-doped AglnS 2 synthesis

$\mathrm{InCl}_{3}(88.4 \mathrm{mg}, 0.4 \mathrm{mmol})$ was added to $\mathrm{AgNO}_{3}(17 \mathrm{mg}, 0.1 \mathrm{mmol})$ in a $100 \mathrm{ml}$ round bottom flask. Subsequently, $190 \mu \mathrm{l}$ of oleic acid $(0.6 \mathrm{mmol}), 720 \mu \mathrm{l}$ of dodecylthiol $(3 \mathrm{mmol})$ 
and $8 \mathrm{ml}$ of octadecene were added under vigorous stirring (420 rpm). The dispersion was flushed with argon for $15 \mathrm{~min}$. Subsequently the temperature was risen and kept at $60^{\circ} \mathrm{C}$ for $15 \mathrm{~min}$, then at $95^{\circ} \mathrm{C}$ for $15 \mathrm{~min}$, and $110^{\circ} \mathrm{C}$ for other $15 \mathrm{~min}$ until dissolution of indium and silver salts. After, $9.6 \mathrm{mg}(0.3 \mathrm{mmol})$ of $\mathrm{S}$ dissolved in $4 \mathrm{ml}$ of oleylamine were rapidly added with a syringe. Immediately, the solution turned from pale pink to dark pink. After 15 min, $70.5 \mathrm{mg}(0.5 \mathrm{mmol})$ of $\mathrm{ZnCl}_{2}$ dissolved in $3 \mathrm{ml}$ of oleylamine and $2 \mathrm{ml}$ of 1 -octadecene were added and the temperature was increased to $150{ }^{\circ} \mathrm{C}$ and kept for other $15 \min$. Then, the reaction was chilled with an ice bath and the crude compound was washed by 6 cycles of washing three times using ethanol and three times using acetone as anti-solvent. Finally, the QDs were dispersed in $20 \mathrm{ml}$ of $\mathrm{CH}_{2} \mathrm{Cl}_{2}$ and stored at $4{ }^{\circ} \mathrm{C}$ (concentration 2 $\mathrm{mg} / \mathrm{ml}$ ). Concentration was calculated evaporating and weighing the solid of a known volume of solution.

\section{Preparation of GO-QDs}

One $\mathrm{mg}$ of QDs was dispersed in $1 \mathrm{mg}$ of $\mathrm{CH}_{2} \mathrm{Cl}_{2}$ in a $20 \mathrm{ml}$ vial. Subsequently, $1 \mathrm{ml}$ of $\mathrm{GO}$ dispersion (1 $\mathrm{mg} / \mathrm{ml}$ in milliQ water) was slowly added with a micropipette allowing the 2phase formation. The vial was capped with a plastic cap pierced with a syringe needle. The dispersion was left under agitation overnight. During this period, the organic phase was completely evaporated. The crude complex was first centrifuged at $1000 \mathrm{rpm}$ for $5 \mathrm{~min}$ to remove unreacted QDs. Subsequently, the supernatant was centrifuged at $13000 \mathrm{rpm}$ for $10 \mathrm{~min}$ and the pellet was re-dispersed in fresh water three times. Control reaction of unreacted GO was also carried out. The supernatant of control GO and GO-QD was analyzed by a spectrophotometer UV-vis (abs $300 \mathrm{~nm}$ ). In case of control reaction, most of the GO remain in the supernatant meanwhile, GO-QD supernatant does not show any appreciable GO signal indicating that the reaction is quantitative for $\mathrm{GO}$. For these reasons we assumed that all the GO has reacted with the QDs. GO-QD was stored at a concentration of $1 \mathrm{mg} \mathrm{GO} / \mathrm{ml}$ in milliQ water in the dark at $4{ }^{\circ} \mathrm{C}$. All the biological experiments have been performed using the same concentration of $\mathrm{GO}$, either pure or in the complex with QDs.

\subsection{Cell culture}

HeLa (epithelial, human cervical adenocarcinoma) and Raw 264.7 (macrophages, Abelson murine leukaemia virus-induced tumour) cells were cultured as mono-layers in Dulbecco's modified Eagle medium supplemented with $10 \mu \mathrm{g} / \mathrm{mL}$ gentamycin (Lonza BioWhittaker), 
$10 \mathrm{mM}$ HEPES (Lonza BioWhittaker), $0.05 \mathrm{mM} \beta$-mercaptoethanol (Lonza BioWhittaker) and $10 \% \mathrm{FBS}$, in a humidified incubator $\left(37^{\circ} \mathrm{C}, 5 \% \mathrm{CO}_{2}\right)$. For toxicity experiments, cells were seeded in 24-well plates (approximately $0.5 \times 10^{5} \mathrm{cells} / \mathrm{cm}^{2}, 1 \mathrm{~mL} / \mathrm{well}$ ). The nanomaterials were diluted in the cell culture media at different concentrations and subsequently cells were exposed for $24 \mathrm{~h}$ to the treatments. For confocal microscopy, the cells were seeded in 8-well chamber slide (Thermo Scientific) (approximately $1 \times 10^{4}$ cells $/ \mathrm{cm}^{2}, 500 \mu \mathrm{l} /$ well). Cells were left to grow until $70-80 \%$ confluency and exposed up to $24 \mathrm{~h}$ to the nanomaterials.

\subsection{Cytotoxicity assay}

Cell viability was determined by flow cytometry using the standard assay with FVD (Fixable Viability Dyes, violet, Invitrogen), $24 \mathrm{~h}$ after exposure to the materials. The supernatant was aspirated and discarded and the cells were washed twice with PBS. Cells were detached from the plates using a scraper in the case of Raw 264.7 and for HeLa was used a solution of $0.25 \%$ Trypsin/ $0.53 \mathrm{mM}$ EDTA. The cells were washed with PBS, $2 \%$ foetal bovine serum and then were incubated (20 min, $4{ }^{\circ} \mathrm{C}$ ) with the dye (dil. 1: 2000). Thereafter, the cells were washed with PBS, $2 \%$ foetal bovine serum and resuspended in $300 \mu \mathrm{l}$ and immediately acquired on the cytometer (Beckman Coulter, Fullerton, CA, USA). At least 10000 cells were counted for each sample, and experiments were performed in triplicate.

\subsection{Data analysis and statistical methods}

Flow cytometry data were generated using FlowJo software (FlowJo LLC, Ashland, OR, USA) and saved as .LMD files. The files were analyzed by FlowJo software version 7.6.5. Cell viability $(\%)$ was calculated as $[(A) /(B) \times 100]$, where $A$ and $B$ are the number of live cells on treated and control groups, respectively. Values represent mean $\pm \operatorname{SD}(n=3)$.

\subsection{Preparation of biological dispersions}

An aliquot of solution corresponding at $1 \mathrm{mg}$ of GO (GO-QD) was centrifuged for $30 \mathrm{~min}$ at $13000 \mathrm{rpm}$. Subsequently, the pellet was re-dispersed in $1.8 \mathrm{ml}$ of DMEM serum free cell media under sterile condition. For "classic" method, $200 \mu \mathrm{l}$ (10\% total volume) of FBS was added dropwise under stirring. For UT method, the GO dispersion was mixed using UltraTurrax at $10000 \mathrm{rpm}$ (1-2 min), while $200 \mu \mathrm{l}$ of FBS (10\% total volume) were added dropwise within 1 to 2 minutes. 


\section{Results and discussions}

\subsection{Material Preparation}

To investigate and validate a new protocol for the preparation of GO dispersions in cell culture medium, we used a material with a size of $600 \pm 300 \mathrm{~nm}$. Fluorescent GO (GOQD) was prepared via functionalization with $\mathrm{ZnS}$ doped $\mathrm{Ag} \ln \mathrm{S}_{2}$ nanocrystals (QDs) with a size of $5 \mathrm{~nm}$ (Figure 1a). The QDs have been synthetized according to the literature ${ }^{14}$ using oleylamine as the capping agent. Tuning the $\mathrm{Zn}$ doping temperature $\left(150{ }^{\circ} \mathrm{C}\right)$, we successfully prepared Far-Red (FR) luminescent nanocrystals with photoluminescence $(\mathrm{PL})$ centered at $720 \mathrm{~nm}$ (Figure 1b).

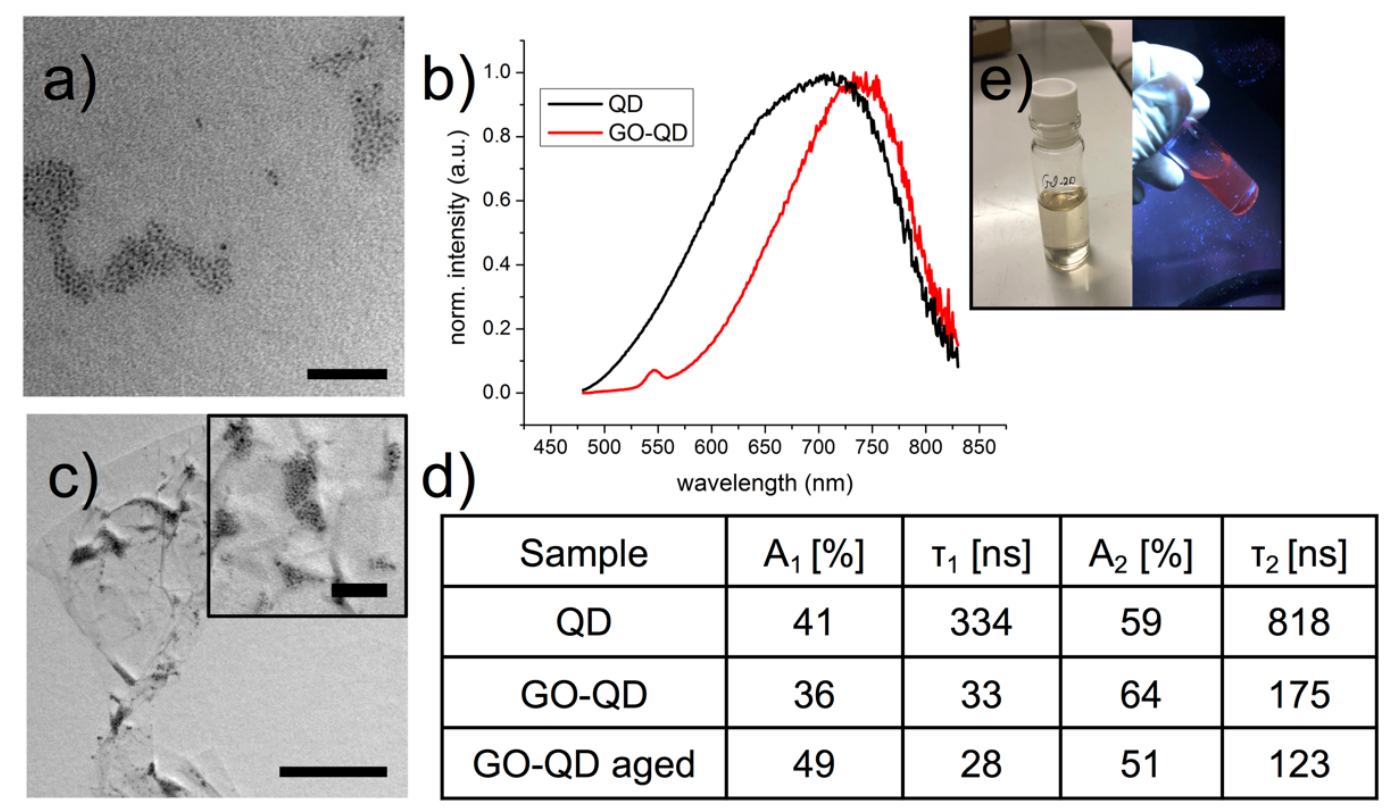

Figure 1. Morphological and photophysical characterization of GO-QD: TEM image of (a) asprepared QDs (Scale bar: $100 \mathrm{~nm}$ ); (b) emission spectra of QDs in $\mathrm{CHCl}_{3}$ and GO-QD in water at $10 \mu \mathrm{g} / \mathrm{ml}\left(\lambda_{\text {ex }} 480 \mathrm{~nm}\right.$ ); (c) TEM image of GO-QD (scale bar: $500 \mathrm{~nm}$ ) [inset: magnification showing QDs onto the GO surface (scale bar $100 \mathrm{~nm}$ )]; (d) lifetime characterization of QDs and GO-QD in $\mathrm{CH}_{2} \mathrm{Cl}_{2}$ and water, respectively. QD dispersion in water was impossible due to their high hydrophobicity; (e) optical image of GO-QD at $10 \mu \mathrm{g} / \mathrm{ml}$ under light and UV lamp.

The complexation of GO with the hydrophobic QDs was performed via simple adsorption. ${ }^{15}$ The GO-QD was obtained through various washing cycles to remove unreacted QDs from the media. Subsequently, the GO-QD was stored in water at $4{ }^{\circ} \mathrm{C}$ in the dark to avoid possible quenching due to photobleaching. TEM analysis (Figure 1c) displayed that the QDs are clearly adsorbed onto the GO surface. In addition, the XPS characterization of GO and QDs are in good agreement with the literature (Figure S1). ${ }^{13,14}$ 
XPS spectrum of GO-QD further supports the coexistence of both GO and QD phases. In addition, functionalization with QD does not alter the size and lateral dimensions of the $G O$ sheets (Figure S2). Steady state fluorescence characterization showed that the GO-QD has an emission centered at $720 \mathrm{~nm}$ (Figure 1b). Lifetime analysis of the QDs in $\mathrm{CH}_{2} \mathrm{Cl}_{2}$ revealed two main contributions: a long emission (818 ns, 59\%) attributed to donoracceptor transition between two localized states inside of the lattice structure and a short emission (334 ns, 41\%) attributed to a surface trap state recombination (Figure 1d). ${ }^{16}$ When QDs are anchored to GO, a sensible decrease of both long (175 ns, 64\%) and short (33 ns, 36\%) contributions was measured. This reduction can be attributed to an energy transfer (ET) process between the QDs and the GO. ${ }^{17}$ The ET is enhanced by the proximity of the $\mathrm{GO}$ to the QDs' donor states and it affects more the luminescence coming from the surface defects of the QDs. Additionally, we investigated the stability of the GOQD adduct after one month aging in water $\left(4{ }^{\circ} \mathrm{C}\right)$. TEM characterization shows that the QDs remain adsorbed onto the GO surface (Figure S3). Lifetime characterization of the aged sample evidenced a negligible change in the lifetime (Figure 1d). This long shelf-life together with the bright luminescence visible to the eye, even at low concentrations (Figure $1 \mathrm{e})$, make the GO-QD an interesting material for bioimaging experiments.

Both $\mathrm{GO}$ and GO-QD tend to form micrometric aggregates as mixed into the complete cell culture media. Stable dispersions can be obtained by mixing the GO or GO-QD with the serum-free cell culture media, but as the serum is added (at a final concentration of $10 \%$ for optimal cell culture conditions) the GO immediately starts to flocculate and it precipitates after $4 \mathrm{~h}$. Similar results have been obtained when fetal bovine serum (FBS) was added under vigorous magnetic stirring. As discussed above, the formation of protein corona involves a covalent and non-covalent interaction between the $\mathrm{GO}$ and the proteins. Furthermore, this process happens very quickly and is completed in a 5-10 min time scale. ${ }^{8}$ Each protein can react or be adsorbed onto more than one $\mathrm{GO}$ sheet, causing the formation of large agglomerates. Considering the fast kinetic of the corona formation, we assumed that the mixing conditions are the key variable to control the agglomeration. The magnetic stirrer often does not go over $900 \mathrm{rpm}$ agitation speed and may be not enough for the $\mathrm{GO}$ and protein fast diffusion in solution. For this reason, we enhanced the stirring conditions using a UT disperser. We decided to set up the UT speed at $10000 \mathrm{rpm}$ to allow an intensive stirring but without inducing damage to the GO or GO-QD flakes (Figure S4). Agglomerate formation has been evaluated by TEM microscopy and the results are shown in Figure 2. 


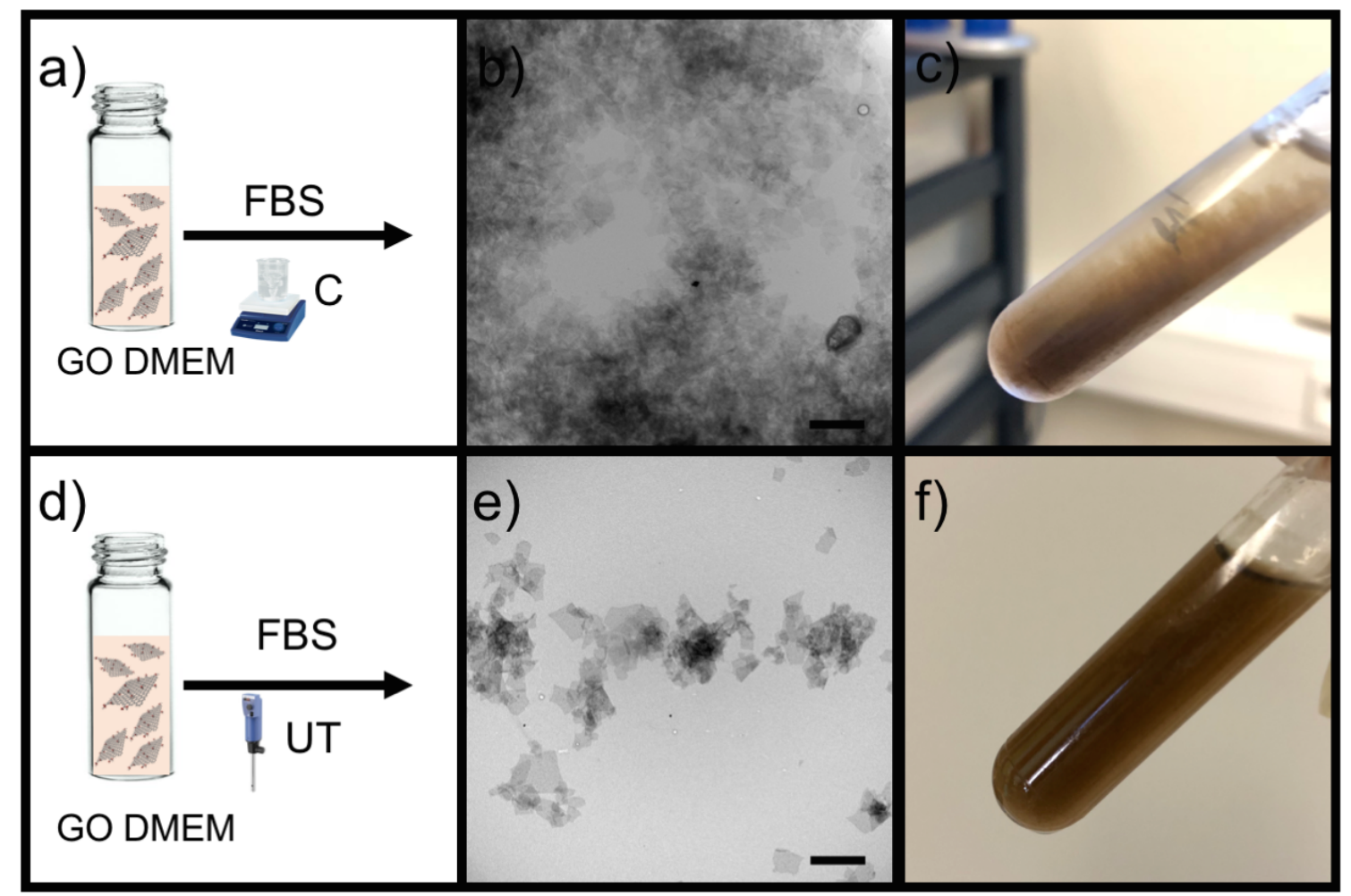

Figure 2. Preparation of $\mathrm{GO}$ for in vitro experiments using the classic (C) or UT protocol in the presence of $10 \%$ FBS: (a) Schematic representation of the preparation of GO C. (b) TEM micrographs of GO C in cell culture media. Scale bar: $2 \mu \mathrm{m}$. (c) Optical image of GO C after $4 \mathrm{~h}$ of incubation. (d) Schematic representation of the preparation of GO UT. (e) TEM micrographs of GO UT in cell culture media. Scale bar: $2 \mu \mathrm{m}$. (f) Optical image of GO UT after $4 \mathrm{~h}$ of incubation.

Classic (C) treatment of GO showed the formation of big agglomerates after contact with FBS (Figure 2b), with sizes larger than $5 \mu \mathrm{m}$. Meanwhile UT treatment for GO displayed a significant decrease of the size of the agglomerates (Figure 2e). This microscopic effect is in agreement with the colloidal stability of the formulations where GO, under classic conditions, leads to a sedimentation after $4 \mathrm{~h}$ (Figure 2c), while the GO prepared via UT has an improved stability and a slower sedimentation (Figure 2f). The mechanism of interaction of GO with proteins in cell culture media is complex and difficult to investigate. As mentioned before, different studies pointed out the fact that $\mathrm{GO}$ can adsorb/react with proteins within 5 to 10 minutes with a high yield (from 2 to 12 times its weight). ${ }^{7,8}$ However, all these studies were generally accomplished using only one protein. FBS is a mixture of elements containing, among others, proteins, amino acids and salts. It is this complexity of elements in the cell culture media that render the elucidation of the exact mechanism of interaction of GO with all the nutrients difficult. Besides, agglomeration of GO is observed when it comes in contact with FBS and this is mainly via an interaction with proteins. ${ }^{18}$ The mechanism of this coronation is mostly the result of a mixture of $\pi-\pi$ interactions and 
epoxide opening reactions. $\pi-\pi$ interactions occur between hydrophobic domains of the proteins and GO, while epoxide opening reactions happen between the epoxide groups of GO and the free amino groups of the protein amino acids. If we focus on GO and protein macrostructures, we can fairly say that each entity possesses more than one active site and so can undergo multiple reactions. Moreover, these active sites react very quickly. GO and proteins are large structures characterized by slow diffusion coefficients, hence the mixing conditions are critical. Therefore, diffusion and coronation rates are crucial for the preparation of a uniform and stable dispersion. It is probable that under "classical" conditions the process of coronation is faster than the $\mathrm{GO}$ and proteins' diffusion velocities. In this case, multiple protein/GO reactions are most likely to happen, leading to the formation of inhomogeneous supramolecular structures composed of multiple sheets and proteins (Figure 2b and c). Instead, the UT, rotating at a speed of $10000 \mathrm{rpm}$, is able to significantly accelerate the GO and protein diffusion rates, fast enough to compete with the complexation/functionalization rate and thus inducing the formation of a stable and homogeneous dispersion (Figure 2e and f).

\subsection{In vitro experiments}

Interaction of GO with the different cell lines was then evaluated using live cell confocal microscopy, which avoids artifacts due to cell fixation. For this purpose, we incubated the cells with GO and GO-QD prepared via "classic" (termed GO C and GO-QD C) or UltraTurrax mixing (termed GO UT and GO-QD UT). The cells were incubated with the material at 5 and $20 \mu \mathrm{g} / \mathrm{ml}$. Sedimentation of the material on top of the cells or in empty spaces of the well can be easily visualized by transmission microscopy after incubation with each material at $20 \mu \mathrm{g} / \mathrm{ml}$ after 4,8 and $24 \mathrm{~h}$ (Figure 3).

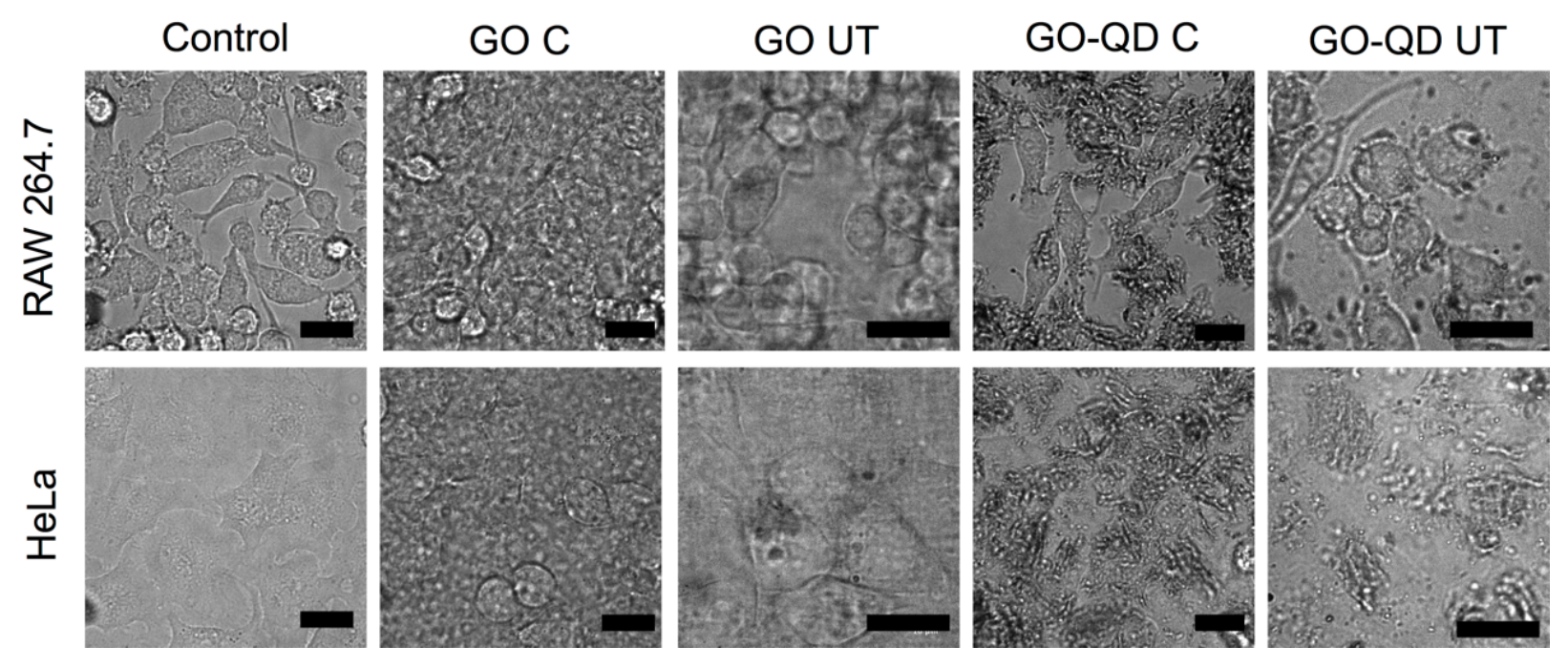


Figure 3. Bright field images of Raw 264.7 (upper panels) and HeLa (lower panels) cells after $24 \mathrm{~h}$ of incubation at $20 \mu \mathrm{g} / \mathrm{ml}$ with the different materials. Scale bar: $10 \mu \mathrm{m}$.

By comparing with the control images (Figure 3, first column from the left), where there is no material at the bottom of the well or on the surface of the cells, we were able to observe how the GO C started its sedimentation at $4 \mathrm{~h}$ and increased it over time (Figure S5). After $24 \mathrm{~h}$ (Figure 3, second column from the left), the GO C sediment has created on both cell lines a thick layer of material that uniformly covers the cell surface and the extracellular space. The same pattern can be observed for GO-QD C (Figure 3, fourth column from the left). In this case the material appears to be thicker, probably due to the high optical density of the QDs on the GO surface.

When the GO is pretreated with UT, the material sedimentation is significantly reduced. Only a few agglomerates were observed after $24 \mathrm{~h}$ in both Raw 264.7 and HeLa cells (Figure 3, third column from the left). GO-QD UT showed a similar behavior (Figure 3, last column). As already reported by Vranic et al., ${ }^{10} \mathrm{GO} \mathrm{C}$ dispersed into cell culture media deposits rapidly on top of the cells forming a thick layer of material. The treatment with UT is instead able to clearly reduce the material agglomeration (Figure $2 \mathrm{~b}$ and $\mathrm{e}$ ) and consequently its sedimentation, improving the colloidal stability in the cell culture media. Subsequently, we were interested in whether the UT treatment was able to influence or modify the uptake and the internalization kinetic of the material compared with the classical method. The internalization was evaluated at a GO-QD concentration of $5 \mu \mathrm{g} / \mathrm{ml}$ at different time points (4, 8 and $24 \mathrm{~h}$ ). We used this low concentration to avoid a thick layer of material on top of the cells that would have made the analysis and interpretation of the results more difficult.

The macrophages treated with the material prepared via the "classic" method showed a low efficiency in the internalization of the material after $24 \mathrm{~h}$ (Figure 4). It is noteworthy to mention that, from the z-stacking analysis, a very low signal was detected inside the cells, while most of the GO-QD C seems stuck in the cell membrane. By $8 \mathrm{~h}$, the UT treatment already allowed the internalization of the material, with a clear localization of the GO-QD UT passing through the cell membrane. 

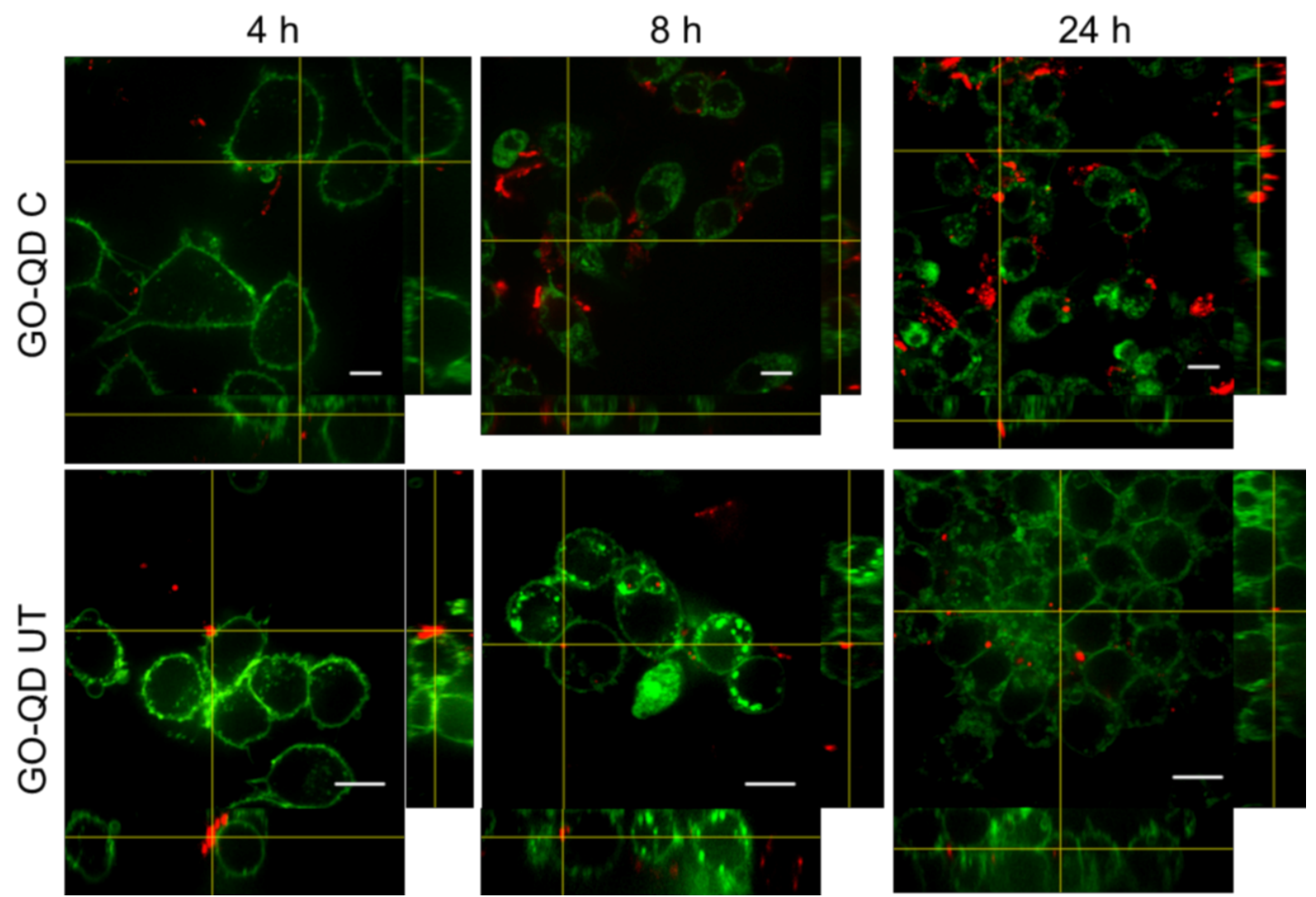

Figure 4. Z-stacking analysis with $x z$ and $y z$ orthogonal views on live Raw 264.7 cells incubated with $5 \mu \mathrm{g} / \mathrm{ml}$ of GO-QD C (upper panel) and UT (lower panel) after 4, 8 and $24 \mathrm{~h}$. In green, membranes stained with Cell-Mask; in red, GO-QDs. Scale bar: $10 \mu \mathrm{m}$.

An analogous pattern was also observed in HeLa cells (Figure 5). GO-QD C uptake was seen after $24 \mathrm{~h}$ of incubation, while in the case of the UT treated sample the material was already internalized after $8 \mathrm{~h}$ of incubation. The uptake efficiency was estimated comparing HeLa and Raw 264.7 cells after incubation with $20 \mu \mathrm{g} / \mathrm{ml}$ of GO-QD after $24 \mathrm{~h}$. In this experiment, we compared the signal coming from different cell height levels by visualizing the plane on top of the cell (external material) and the plane in the middle (internalized material) (Figure S6). 

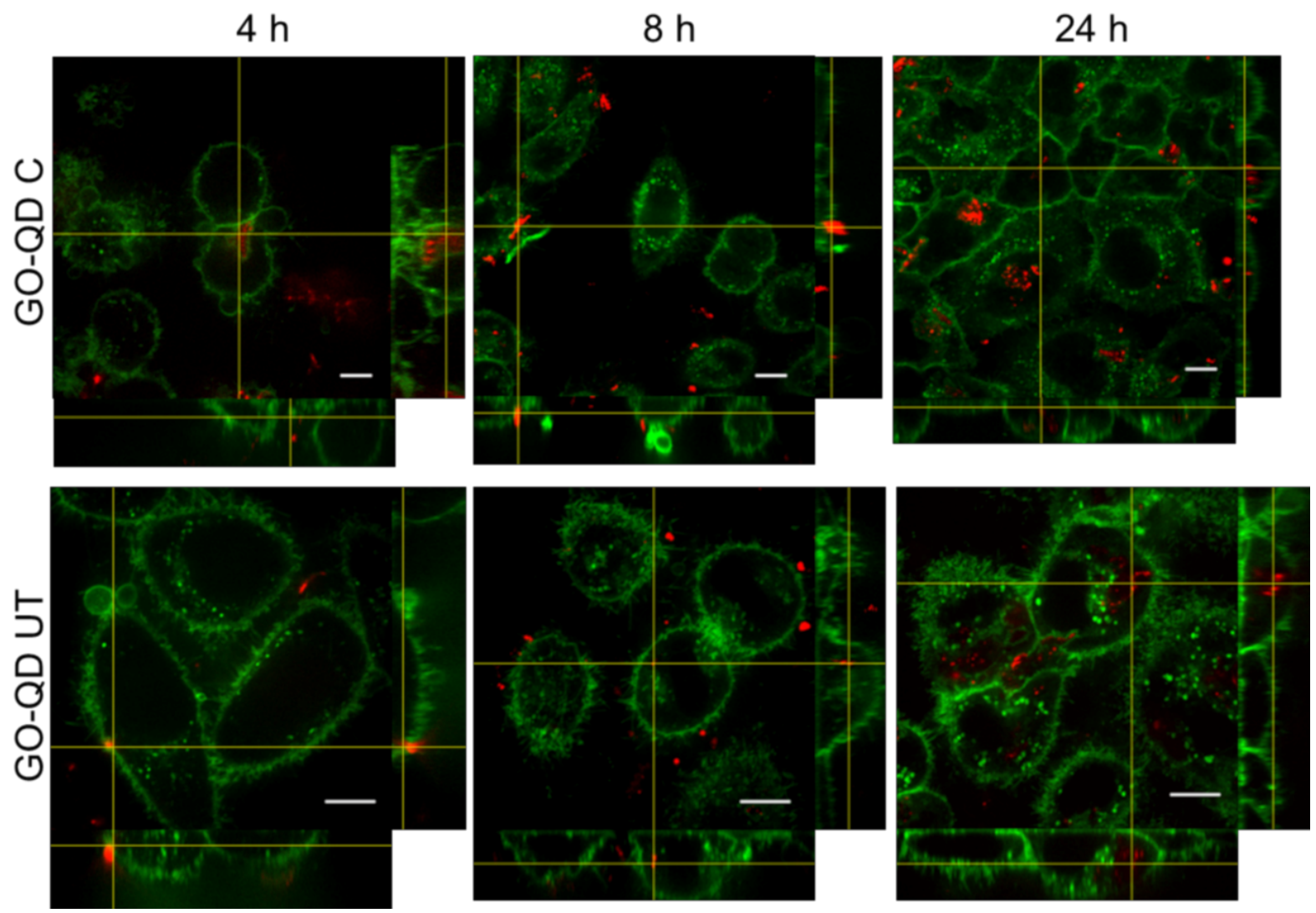

Figure 5. Z-stacking analysis with $x z$ and $y z$ orthogonal views on live HeLa cells incubated with 5 $\mu \mathrm{g} / \mathrm{ml}$ of GO-QD C (upper panel) and UT (lower panel) after 4, 8 and $24 \mathrm{~h}$. In green, membranes stained with Cell-Mask; in red, GO-QD. Scale bar: $10 \mu \mathrm{m}$.

GO-QD prepared with the "classic" procedure is localized mostly on top of Raw 264.7 and HeLa cells (Figure 6). The analysis of the middle planes showed that a small amount of material is inside the cells, while most of the GO-QD C is localized on the cell surface or in the extracellular space. In the case of GO-QD UT, only a few aggregates were detected on top of the cells (Figure 6), while a significant accumulation of the GO-QD UT was observed inside cell membranes. Similar results were obtained with HeLa cells. This remarkable difference in uptake efficiency can also be appreciated in Video S1 and Video S2 (GO-QD C and GO-QD UT) for Raw 264.7 cells and in Video S3 and Video S4 (GO-QD C and GOQD UT) for HeLa cell lines. 

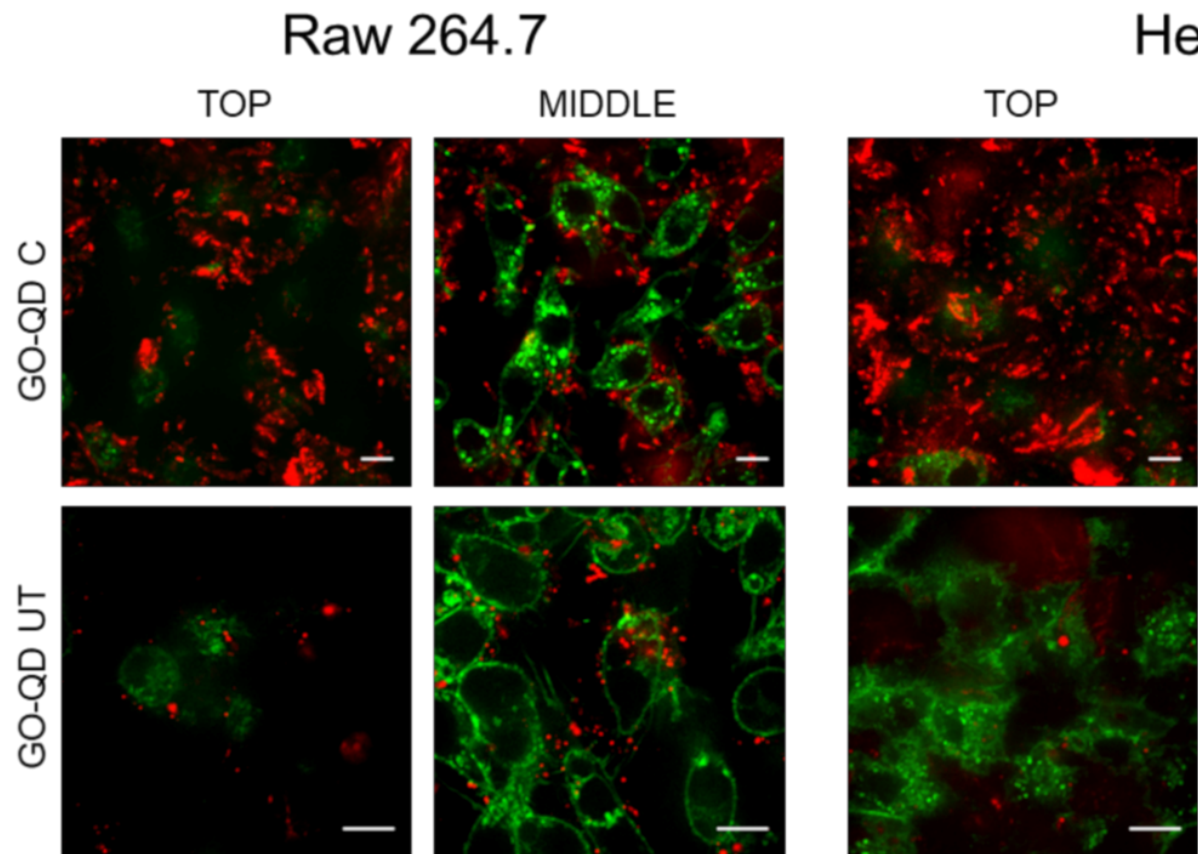

HeLa
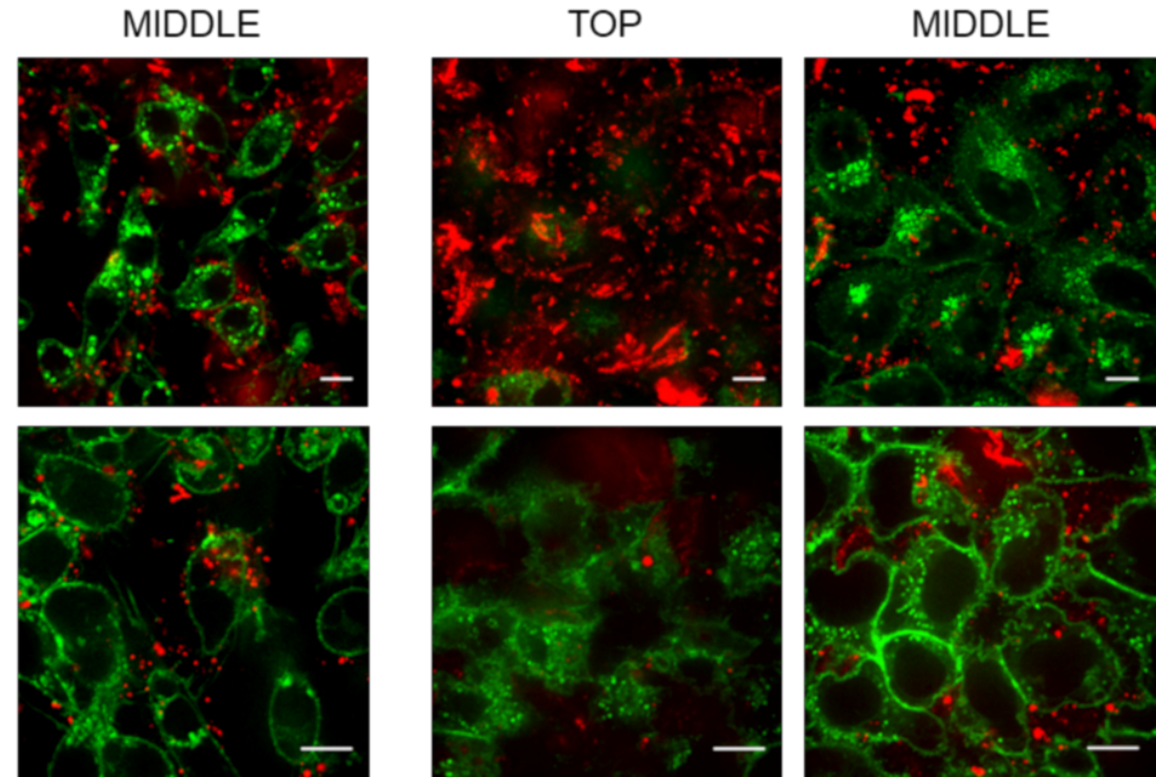

Figure 6. Interaction of GO-QD with Raw 264.7(left panel) and Hela (right panel) cells. Comparison between the surface height top level of the cells (TOP columns) and the middle height level (MIDDLE columns). In green, membranes stained with Cell-Mask; in red, GO-QD. Scale bar: $10 \mu \mathrm{m}$.

The full assessment of this protocol consisted also on the study of the cytotoxic effect of GO-based materials on the cells treated in the different conditions.

In vitro toxicological characterization of the GO and GO-QD prepared by both methods was evaluated through the degree of cell survival by flow cytometry using the standard assay with violet Fixable Viability Dye (FVD). The analysis of cytotoxicity after incubation in HeLa cells with the nanomaterials showed that viability was not significantly reduced by the presence of the GO or GO-QD up to $100 \mu \mathrm{g} / \mathrm{ml}$ concentration after $24 \mathrm{~h}$ of treatment (Figure $7 a, b)$. In the case of Raw 264.7 the viability is significantly reduced to $40 \%$ for the cells treated with GO at the highest concentrations. Regarding the GO preparation method, we did not observe highly significant viability differences except at the lowest concentration $(5 \mu \mathrm{g} / \mathrm{ml})$. In this latter case, GO prepared by ultra-mixing could be used to satisfactory achieve adequate intracellular concentrations of the material while avoiding significant cell damage.

In the case of GO-QD, the material obtained with the UT method improved the viability up to $80-100 \%$ compared to the control, while with the classic method we obtained similar results to GO. These data are in agreement with different studies where cell viability 
decreases by $20 \%$ after exposure to $\mathrm{GO}$ concentrations around $10 \mu \mathrm{g} / \mathrm{ml}$ during $24 \mathrm{~h}$ or longer. ${ }^{19}$

a)

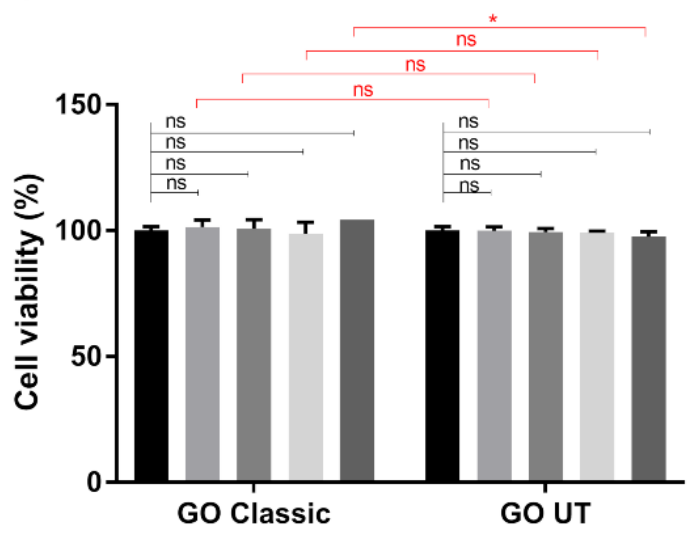

c)

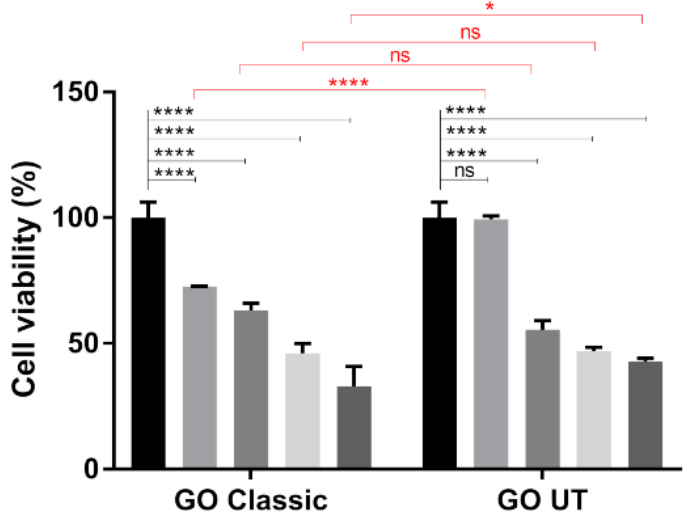

b)

HeLa

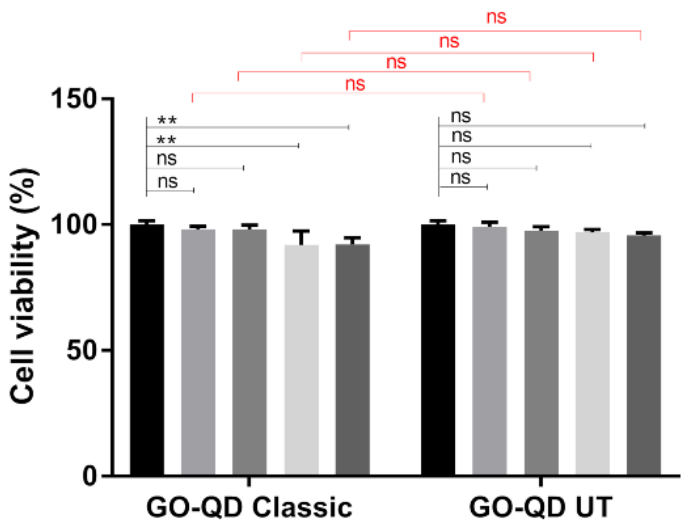

d)

Raw 264.7

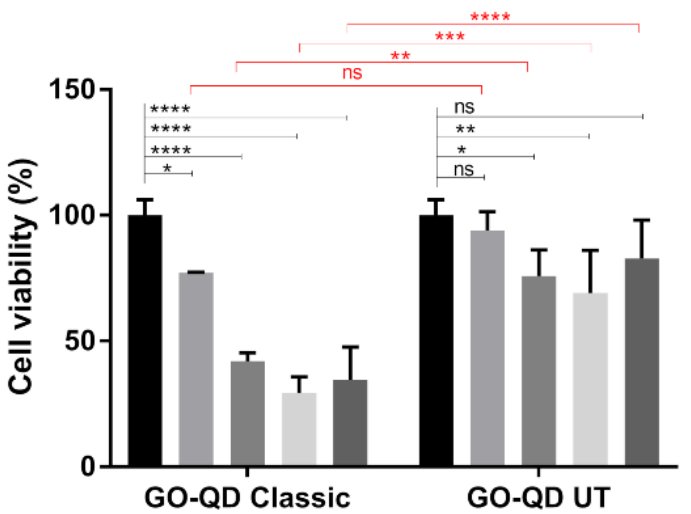

- Control $-5 \mu \mathrm{g} / \mathrm{ml}=25 \mu \mathrm{g} / \mathrm{ml}=50 \mu \mathrm{g} / \mathrm{ml}=100 \mu \mathrm{g} / \mathrm{ml}$

Figure 7. Evaluation of cell viability by flow cytometry using the standard assay with violet FVD. (a, b) HeLa cells. (c, d) Raw 264.7 cells. Cells were treated with the nanomaterials prepared by both methods ( $C$ and UT) for $24 \mathrm{~h}$. Data represent means $\pm S D(n=3)$. * (in black) shows statistically significance compared with the control. * (in red) shows statistically significance comparing the method used in the preparation of the nanomaterial (Two-way ANOVA followed by Bonferroni's post-test: ns $p>0.05,{ }^{*} p<0.05,{ }^{* *} p<0.01$, $\left.{ }^{* * *} p<0.001,{ }^{* * * *} p<0.0001\right)$.

To analyze how the GO and GO-QD affect cell viability, we need to consider how the cells "see" the nanomaterials depending on their own characteristics. The differences between the composition of the cell membrane in HeLa and Raw 264.7 cells, including their uptake 
mechanisms and translocation, will influence the nanomaterial's fate in the intracellular environment, since transport greatly depends on the composition of cellular membranes. It is likely that the toxicity may be induced by the interaction of the nanomaterials with cells' membranes in Raw 264.7 (i.e., professional phagocytes). This hypothesis is also corroborated by the FVD standard assay, where the reactive dye can permeate the damaged membranes of dead cells and covalently attaches to the interior and exterior amines of the proteins. This result highlights the importance of evaluating nano-safety by using different cellular systems. The observed sensitivity of Raw 264.7 macrophages, among other immune cells, compared with epithelial cell lines has been described in the design of comprehensive strategies in in vitro toxicity testing of a panel of representative oxide nanomaterials. ${ }^{20}$

Furthermore, the physic-chemical characteristics of the nanomaterials, like composition, size, dispersibility and how they interact with the biological components of the medium in which they are dispersed, also influences the cytotoxicity. As a result of the coronation process of the material with the proteins of the cell culture medium, its composition, which is essential for cell nutrition, can be significantly changed leading to non-optimal cell culture conditions for a cell line.

Size, shape and agglomeration of the nanomaterials have been also found to greatly influence their cellular uptake. In our case, classic mixing and UT mixing could play an important role in the speed, quantity and mechanism of the nanomaterials taken up by macrophages and HeLa cells. They might aggregate into many different shapes and sizes that make it hard to dictate the outcome and interpretation of the results regarding internalization mechanisms. By only analyzing the aggregate's size, the internalization pathway change. ${ }^{21,22}$ In addition, it has been shown how coronation is able to sensibly affect the cytotoxicity and the uptake mechanism. ${ }^{6,22} \mathrm{~A}$ preliminary evidence about the possible internalization mechanism by RAW 264.7 can be inferred from Figure S7. In the case of GO-QD C the material appears trapped into the cell membranes (Figure S7a and b), while UT treatment shows the internalization by formation of membrane protrusions, allowing the uptake of the material likely via macropinocytosis, a mechanism characteristic of this type of phagocytic cells (Figure S7c-e). GO-QD UT are also clearly visible inside endocytic vesicles (Figure S7f-i). For aggregates in the range of a few hundred nanometers, the predictable behavior would be clathrin-mediated endocytosis, while aggregates $>1 \mu \mathrm{m}$ are trapped into large fluid pockets by formation and enclosure of 
membrane protrusions. ${ }^{21}$ Our results correlate with the data already reported in the literature. ${ }^{22}$

Toxicity of nanomaterials and in particular the graphene family is a complex field. ${ }^{23}$ Unlike soluble drugs, where concentration and exposure time are the most important parameters, graphene oxide guidelines for toxicity regards also shape, ${ }^{24}$ size, ${ }^{25,26}$ surface functionalization $^{27,28}$ and number of layers. ${ }^{29,30}$ For instance, we have already demonstrated how GO size, oxidation degree and functionalization play an important role in the denaturation of siRNA. ${ }^{31}$ Moreover, the general toxicity depends on the cell and animal models used, where in some cases it can be harmful ${ }^{2}$ and in others it can induce genotoxicity ${ }^{25}$ and/ or long term side effects. ${ }^{32}$ Considering this information, the answer to the toxicity of GO or GO-QD is not straightforward and would require more study including in vivo tests. The aim of this study was to describe and propose a new general protocol to obtain well-dispersed GO samples in cell culture media for cell imaging and tracking. In this context, we would like to underline the importance of producing colloidal stable nanomaterial dispersions. Highly polydisperse formulations would certainly lead to less predictive and irreproducible results due to the intrinsic inhomogeneity of the systems. The novelty of the proposed protocol is certainly the mild mixing conditions, which does not involve the use of any surfactant or stabilizers. Therefore, it can virtually be applied to all GO formulations and can be used as standard for the new generation of graphene-based drug delivery systems.

\section{Conclusions}

In conclusion, our results using the UT confirm the importance of assuring high dispersibility during protein corona formation in the preparation of a nanomaterial for biomedical applications. Smaller aggregates and a more homogeneous protein corona contribute not only to improve colloidal stability of the formulation but also to mitigate cytotoxicity and change the biological responses to the material in terms of cellular uptake and translocation. We believe that the standardization of the UT method in the preparation of GO-based materials for biological applications will be of great interest to the nano-safety and nano-pharmaceutical scientific community to improve cell viability and uptake efficiency of GO-based materials. 


\section{Acknowledgments}

The authors gratefully acknowledge the financial support from the EU GRAPHENE Flagship project (no. 696656 and no. 785219), from the Agence Nationale de la Recherche (ANR) through the LabEx project Chemistry of Complex Systems (ANR-10LABX-0026_CSC). This work was partly supported by the Centre National de la Recherche Scientifique (CNRS), the International Center for Frontier Research in Chemistry (icFRC), the JST PRESTO, and the JSPS KAKENHI (Science of Atomic Layers (SATL), Grant number 16H00915). The authors thank S. Bernacchi for help in fluorescence measurements, and C. Royer and V. Demais for TEM analysis at the Plateforme Imagerie in Vitro at the Center of Neurochemistry (Strasbourg, France).

Supporting Information. Additional physic-chemical characterization of the nanomaterials (XPS analysis and TEM micrographs) and cell imaging are available free of charge on the ACS Publications website at DOI:

\section{Author Information.}

\section{Corresponding Authors}

*E-mail: a.bianco@ibmc-cnrs.unistra.fr (A.B.)

*E-mail: g.reina@ibmc-cnrs.unistra.fr (G.R.)

\section{ORCID}

Giacomo Reina: 0000-0002-6399-1783

Amalia Ruiz: 0000-0001-8358-5304

Yuta Nishina

Alberto Bianco: 0000-0002-1090-296X

\section{Funding}

\section{References}

(1) Qu, Y.; He, F.; Yu, C.; Liang, X.; Liang, D.; Ma, L.; Zhang, Q.; Lv, J.; Wu, J. Advances on Graphene-Based Nanomaterials for Biomedical Applications. Mater. Sci. Eng. C 2018, 90, 764-780.

(2) Reina, G.; González-Domínguez, J. M.; Criado, A.; Vázquez, E.; Bianco, A.; Prato, 
M. Promises, Facts and Challenges for Graphene in Biomedical Applications. Chem.Soc. Rev. 2017, 46, 4400-4416.

(3) Makharza, S.; Cirillo, G.; Bachmatiuk, A.; Ibrahim, I.; Ioannides, N.; Trzebicka, B.; Hampel, S.; Rümmeli, M. H. Graphene Oxide-Based Drug Delivery Vehicles: Functionalization, Characterization, and Cytotoxicity Evaluation. J. Nanoparticle Res. 2013, 15, 2099.

(4) de Melo-Diogo, D.; Lima-Sousa, R.; Alves, C. G.; Costa, E. C.; Louro, R. O.; Correia, I. J. Functionalization of Graphene Family Nanomaterials for Application in Cancer Therapy. Colloids Surf. B 2018, 171, 260-275.

(5) Ou, L.; Song, B.; Liang, H.; Liu, J.; Feng, X.; Deng, B.; Sun, T.; Shao, L. Toxicity of Graphene-Family Nanoparticles: A General Review of the Origins and Mechanisms. Part. Fibre Toxicol. 2016, 13, 57.

(6) Duan, G.; Kang, S.; Tian, X.; Garate, J. A.; Zhao, L.; Ge, C.; Zhou, R. Protein Corona Mitigates the Cytotoxicity of Graphene Oxide by Reducing Its Physical Interaction with Cell Membrane. Nanoscale 2015, 7, 15214-15224.

(7) Sopotnik, M.; Leonardi, A.; Križaj, I.; Dušak, P.; Makovec, D.; Mesarič, T.; Ulrih, N. P.; Junkar, I.; Sepčić, K.; Drobne, D. Comparative Study of Serum Protein Binding to Three Different Carbon-Based Nanomaterials. Carbon 2015, 95, 560-572.

(8) Chong, Y.; Ge, C.; Yang, Z.; Garate, J. A.; Gu, Z.; Weber, J. K.; Liu, J.; Zhou, R. Reduced Cytotoxicity of Graphene Nanosheets Mediated by Blood-Protein Coating. ACS Nano 2015, 9, 5713-5724.

(9) Xu, X.; Mao, X.; Wang, Y.; Li, D.; Du, Z.; Wu, W.; Jiang, L.; Yang, J.; Li, J. Study on the Interaction of Graphene Oxide-silver Nanocomposites with Bovine Serum Albumin and the Formation of Nanoparticle-Protein Corona. Int. J. Biol. Macromol. 2018, 116, 492-501.

(10) Vranic, S.; Rodrigues, A. F.; Buggio, M.; Newman, L.; White, M. R. H.; Spiller, D. G.; Bussy, C.; Kostarelos, K. Live Imaging of Label-Free Graphene Oxide Reveals Critical Factors Causing Oxidative-Stress-Mediated Cellular Responses. ACS Nano 2018, 12, 1373-1389.

(11) $\mathrm{Hu}, \mathrm{X} . ; \mathrm{Li}, \mathrm{D} . ; \mathrm{Mu}, \mathrm{L}$. Biotransformation of Graphene Oxide Nanosheets in Blood Plasma Affects Their Interactions with Cells. Environ. Sci. Nano 2017, 4, 15691578.

(12) Hu, W.; Peng, C.; Lv, M.; Li, X.; Zhang, Y.; Chen, N.; Fan, C.; Huang, Q. Protein Corona-Mediated Mitigation of Cytotoxicity of Graphene Oxide. ACS Nano 2011, 5 , 
3693-3700.

(13) Morimoto, N.; Kubo, T.; Nishina, Y. Tailoring the Oxygen Content of Graphite and Reduced Graphene Oxide for Specific Applications. Sci. Rep. 2016, 6, 21715.

(14) Wang, X.; Xie, C.; Zhong, J.; Liang, X.; Xiang, W. Synthesis and Temporal Evolution of Zn-Doped AgInS2 Quantum Dots. J. Alloys Compd. 2015, 648, 127-133.

(15) Sheng, Y.; Tang, X.; Peng, E.; Xue, J. 3. J. Mater. Chem. B 2013, 1, 512-521.

(16) Mao, B.; Chuang, C.-H.; McCleese, C.; Zhu, J.; Burda, C. Near-Infrared Emitting AgInS2/ZnS Nanocrystals. J. Phys. Chem. C 2014, 118, 13883-13889.

(17) Morales-Narváez, E.; Pérez-López, B.; Pires, L. B.; Merkoçi, A. Simple Förster Resonance Energy Transfer Evidence for the Ultrahigh Quantum Dot Quenching Efficiency by Graphene Oxide Compared to Other Carbon Structures. Carbon 2012, 50, 2987-2993.

(18) Mukherjee, S. P.; Bottini, M.; Fadeel, B. Graphene and the Immune System: A Romance of Many Dimensions. Front. Immunol. 2017, 8, 673.

(19) Pinto, A. M.; Gonçalves, I. C.; Magalhães, F. D. Graphene-Based Materials Biocompatibility: A Review. Colloids Surfaces B Biointerfaces 2013, 111, 188-202.

(20) Farcal, L.; Torres Andón, F.; Di Cristo, L.; Rotoli, B. M.; Bussolati, O.; Bergamaschi, E.; Mech, A.; Hartmann, N. B.; Rasmussen, K.; Riego-Sintes, et al. Comprehensive In Vitro Toxicity Testing of a Panel of Representative Oxide Nanomaterials: First Steps towards an Intelligent Testing Strategy. PLoS One 2015, 10 (5), e0127174.

(21) Verma, A.; Stellacci, F. Effect of Surface Properties on Nanoparticle-Cell Interactions. Small 2010, 6, 12-21.

(22) Mu, Q.; Su, G.; Li, L.; Gilbertson, B. O.; Yu, L. H.; Zhang, Q.; Sun, Y.-P.; Yan, B. Size-Dependent Cell Uptake of Protein-Coated Graphene Oxide Nanosheets. ACS Appl. Mater. Interfaces 2012, 4 (4), 2259-2266.

(23) Fadeel, B.; Bussy, C.; Guijarro, S. M.; Vazquez, E.; Flahaut, E.; Mouchet, F.; Evariste, L.; Gauthier, L.; Koivisto, J.; Vogel, U.; et al. Safety Assessment of Graphene-Based Materials: Focus on Human Health and the Environment. ACS Nano 2018, 12, 10582-10620.

(24) Akhavan, O.; Ghaderi, E.; Emamy, H.; Akhavan, F. Genotoxicity of Graphene Nanoribbons in Human Mesenchymal Stem Cells. Carbon 2013, 54, 419-431.

(25) Akhavan, O.; Ghaderi, E.; Akhavan, A. Size-Dependent Genotoxicity of Graphene Nanoplatelets in Human Stem Cells. Biomaterials 2012, 33, 8017-8025.

(26) Lalwani, G.; D’Agati, M.; Khan, A. M.; Sitharaman, B. Toxicology of Graphene-Based 
Nanomaterials. Adv. Drug Deliv. Rev. 2016, 105, 109-144.

(27) Akhavan, O.; Ghaderi, E.; Esfandiar, A. Wrapping Bacteria by Graphene Nanosheets for Isolation from Environment, Reactivation by Sonication, and Inactivation by Near-Infrared Irradiation. J. Phys. Chem. B 2011, 115, 6279-6288.

(28) Li, R.; Guiney, L. M.; Chang, C. H.; Mansukhani, N. D.; Ji, Z.; Wang, X.; Liao, Y.-P.; Jiang, W.; Sun, B.; Hersam, M. C.; et al. Surface Oxidation of Graphene Oxide Determines Membrane Damage, Lipid Peroxidation, and Cytotoxicity in Macrophages in a Pulmonary Toxicity Model. ACS Nano 2018, 12, 1390-1402.

(29) Akhavan, O.; Ghaderi, E. Toxicity of Graphene and Graphene Oxide Nanowalls Against Bacteria. ACS Nano 2010, 4, 5731-5736.

(30) Liao, C.; Li, Y.; Tjong, S.; Liao, C.; Li, Y.; Tjong, S. C. Graphene Nanomaterials: Synthesis, Biocompatibility, and Cytotoxicity. Int. J. Mol. Sci. 2018, 19, 3564.

(31) Reina, G.; Chau, N. D. Q.; Nishina, Y.; Bianco, A. Graphene Oxide Size and Oxidation Degree Govern Its Supramolecular Interactions with SiRNA. Nanoscale 2018, 10 5965-5974.

(32) Akhavan, O.; Ghaderi, E.; Hashemi, E.; Akbari, E. Dose-Dependent Effects of Nanoscale Graphene Oxide on Reproduction Capability of Mammals. Carbon N. Y. 2015, 95, 309-317. 